\title{
Evolution of Severe Aplastic Anemia to Myelodysplasia with Monosomy 7 Following Granulocyte Colony-Stimulating Factor, Erythropoietin and High-Dose Methylprednisolone Combination Therapy
}

\author{
Akimichi OHsaka, Yuichi Sugahara, Yasufumi IMAI and Masahiro KIKUCHI*
}

\begin{abstract}
A 19-year-old man was diagnosed as having severe aplastic anemia and received high-dose methylprednisolone treatment without hematological response. A second course of high-dose mPSL treatment together with granulocyte colony-stimulating factor (G-CSF) plus erythropoietin (EPO) was then started and resulted in trilineage blood cell response. Ten months after the combination therapy thrombocytopenia developed and cytogenetic analysis showed $45, \mathrm{XX},-7$, indicating an evolution to myelodysplastic syndrome (MDS) associated with monosomy 7. G-CSF and EPO treatment together with immunosuppression may be an effective therapy in SAA patients, but such a therapy may increase the risk of evolution to MDS.
\end{abstract}

(Internal Medicine 34: 892-895, 1995)

Key words: trilineage response, myelodysplastic syndrome, clonal evolution

\section{Introduction}

Aplastic anemia (AA) is characterized by refractory cytopenia and bone marrow hypocellularity. Survival of patients with severe disease (SAA) has improved since bone marrow transplantation (BMT) and immunosuppressive treatment with antilymphocyte or antithymocyte globulin became available (1). SAA patients who cannot undergo BMT and who fail to respond to standard therapy show a high mortality rate from complications related to pancytopenia. Granulocyte colonystimulating factor (G-CSF) has recently been used to treat AA patients with some improvement in the peripheral blood neutrophil count (2). We reported previously that G-CSF enhances the superoxide release in neutrophils from AA patients as well as from normal neutrophils (3). These findings suggest that GCSF enhances the host defense against microorganisms both by increasing circulating neutrophil numbers and by enhancing neutrophil functions in AA patients. However, the risks of such therapy in AA patients are not well known. We describe here an SAA patient who was successfully treated with combination therapy consisting of G-CSF, erythropoietin (EPO) and highdose methylprednisolone (mPSL), however subsequently developed myelodysplastic syndrome (MDS) associated with monosomy 7.

\section{Case Report}

A 19-year-old man was admitted to our hospital in April 1993 because of fatigue and exertional dyspnea. On admission he was pale but had no organomegaly. Hematological findings were hemoglobin $(\mathrm{Hb}) 6.2 \mathrm{~g} / \mathrm{dl}$; reticulocytes $13.2 \times 10^{9} / l$; platelets $25 \times 10^{9} / l$; and white blood cells (WBC) $4.9 \times 10^{9} / l$ with $24 \%$ neutrophils, $2 \%$ eosinophils, $3 \%$ monocytes and $71 \%$ lymphocytes. A bone marrow aspirate and biopsy revealed marked hypocellularity without proliferation of blasts and dysplastic changes in myeloid, erythroid and megakaryocytic lineages (Table 1). The karyotype was normal in 20/20 metaphase preparations of bone marrow cells. The serum iron was $305 \mu \mathrm{g} / \mathrm{dl}$, unsaturated iron binding capacity $17 \mu \mathrm{g} / \mathrm{dl}$ and ferritin $788 \mathrm{ng} / \mathrm{dl}$. Direct and indirect Coombs tests, Ham test and sugar-water test were negative. The plasma concentrations of G-CSF and EPO were $110 \mathrm{pg} / \mathrm{ml}$ and $3,740 \mathrm{mU} / \mathrm{ml}$, respectively. Subsequent platelet counts decreased to less than $20 \times 10^{9} /$ l. The patient was thus diagnosed as having SAA (4).

In the absence of an HLA-match sibling donor for BMT he was treated with bolus injections of mPSL $(1,000 \mathrm{mg}$ for 3 days), followed by tapering with oral prednisolone. There was no hematological response, and he remained transfusion-dependent (Fig. 1). In June 1993, he received G-CSF (300 $\mu \mathrm{g}$

From the Department of Internal Medicine, Division of Hematology and *the Department of Pediatrics, Hitachi General Hospital, Ibaraki Received for publication January 6, 1995; Accepted for publication May 31, 1995

Reprint requests should be addressed to Dr. Akimichi Ohsaka, the Department of Internal Medicine, Division of Hematology, Hitachi General Hospital, 2-1-1 Jonan-cho, Hitachi, Ibaraki 317 
Table 1. Bone Marrow Findings during the Course of the Disease

\begin{tabular}{lccc}
\hline Date & $4 / 1993$ & $7 / 1994$ & $8 / 1994$ \\
\hline NCC $\left(\times 10^{9} / l\right)$ & 1.9 & 17.1 & 11.9 \\
Megakaryocytes $\left(\times 10^{6} / l\right)$ & 0 & 16 & 16 \\
Erythroid $(\%)$ & 10.9 & 50.4 & 55.4 \\
Myeloid $(\%)$ & & & \\
Myeloblasts & 0.1 & 1.9 & 2.3 \\
Promyelocytes & 0.6 & 0.7 & 0.6 \\
Myelocytes & 3.8 & 5.1 & 5.4 \\
Metamyelocytes & 6.1 & 5.1 & 5.4 \\
Band neutrophils & 6.0 & 6.4 & 4.8 \\
Segmented neutrophils & 7.5 & 8.7 & 6.6 \\
Eosinophils & 2.9 & 1.9 & 1.0 \\
Basophils & 0.3 & 0.4 & 0.4 \\
$\quad$ Monocytes & 2.4 & 2.8 & 1.1 \\
Lymphoid $(\%)$ & & & 13.8 \\
Lymphocytes & 54.5 & 14.6 & 0.1 \\
Plasma cells & 3.6 & 0.4 & - \\
Dysmegakaryopoiesis* & $\mathrm{NE}$ & - & + \\
Dyserythropoiesis* & - & \pm & - \\
Dysgranulopoiesis* & - & $46, \mathrm{XY}(10 / 13)$ & $46, \mathrm{XY}(2 / 20)$ \\
Chromosome analysis & $46, \mathrm{XY}(20 / 20)$ & $\mathrm{XY},-7(3 / 13)$ & $45, \mathrm{XY},-7(18 / 20)$ \\
& & & \\
\hline
\end{tabular}

*NE: not evaluable, - absent, \pm mild, + moderate, NCC: nucleated cell count.

daily) and EPO (12-18 $\times 10^{3} \mathrm{U}$ daily) treatment simultaneously for 2 weeks. The doses of G-CSF and EPO were elevated to 600 $\mu \mathrm{g}$ and $24 \times 10^{3} \mathrm{U}$, respectively, because of the absence of marked hematological responses. Four weeks after the initiation of hemopoietic growth factor treatment, absolute neutrophil counts were increased but platelet counts and $\mathrm{Hb}$ levels were still low. Thus, he received a second course of high-dose mPSL treatment together with G-CSF and EPO treatment. Two weeks after this combination therapy, evidence of trilineage blood cell responses became apparent, and red cell and platelet transfusions were no longer required (Fig. 1). After cessation of hemopoietic growth factor treatment, he received oxymetholone (20 mg daily) orally for maintenance treatment. In September 1993, he was discharged to our outpatient clinic and was well for 10 months.

In July 1994, the patient was admitted again to our hospital because of fever and purpura. Hematological findings were $\mathrm{Hb}$ $13.4 \mathrm{~g} / \mathrm{dl}$; platelets $3 \times 10^{9} / l$; and WBC $2.8 \times 10^{9} / l$ with $45 \%$ neutrophils, $13 \%$ monocytes and $42 \%$ lymphocytes. A bone marrow aspirate showed normocellularity and erythroid hyperplasia with mild dyserythropoiesis, i.e., abnormal nuclear shape (Table 1). Antibiotic therapy and G-CSF administration (300$600 \mu \mathrm{g}$ daily) were started. Two weeks after the initiation of GCSF, absolute neutrophil counts were increased but platelet counts were still low. He received high-dose mPSL treatment together with G-CSF administration without success. A bone marrow aspirate revealed $2.3 \%$ blasts and erythroid hyperplasia with dysplastic changes in erythroid lineage but not in myeloid and megakaryocytic lineages (Table 1). Cytogenetic analysis of bone marrow cells showed 45,XY,-7 in 18/20 metaphases analyzed (Fig. 2). Retrospectively, cytogenetic analysis on readmission also showed $45, \mathrm{XY},-7$ in 3/13 metaphases analyzed. Thus, evolution to MDS associated with monosomy 7 was diagnosed. In October 1994, he was discharged and has been processed for BMT from unrelated donors.

\section{Discussion}

We presented a patient with SAA who was successfully treated with a combination therapy consisting of G-CSF, EPO and high-dose mPSL, but subsequently developed MDS associated with monosomy 7 . The first course of high-dose mPSL treatment alone showed no hematological response. However, the second course of high-dose mPSL treatment together with G-CSF and EPO treatment resulted in a trilineage response. GCSF therapy alone has been reported to increase neutrophil counts without improvement in platelet counts or Hb levels in children with SAA (2). Simultaneous administration of G-CSF and EPO has resulted in trilineage response in SAA patients, but long-term administration (more than 14 months) was required for such improvement (5). Furthermore, combination therapy with G-CSF and cyclosporin A with or without EPO achieved trilineage responses in SAA patients $(6,7)$. The mechanism of this effect remains to be determined. G-CSF has been reported to enhance the proliferation of hemopoietic stem cells (8). Indeed, prolonged administration of G-CSF can mobilize 


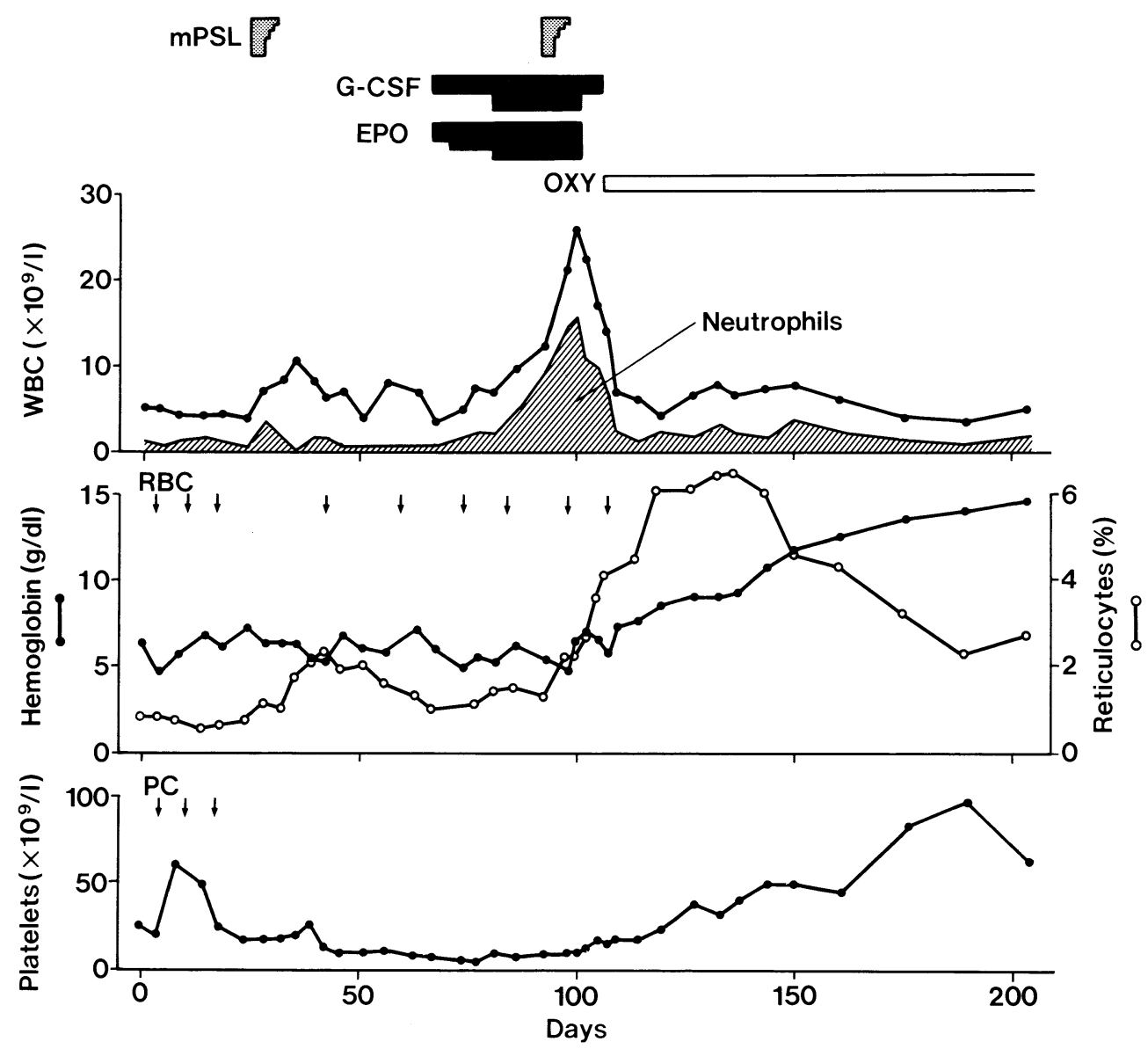

Figure 1. Clinical course of the patient. mPSL: methylprednisolone, G-CSF: granulocyte colony-stimulating factor, EPO: erythropoietin, OXY: oxymetholone.

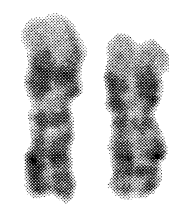

1

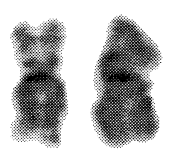

6

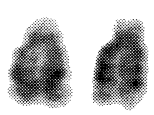

13

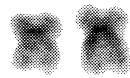

19

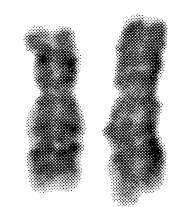

2

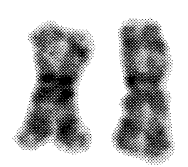

3

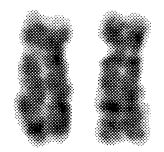

4

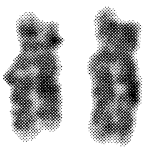

5

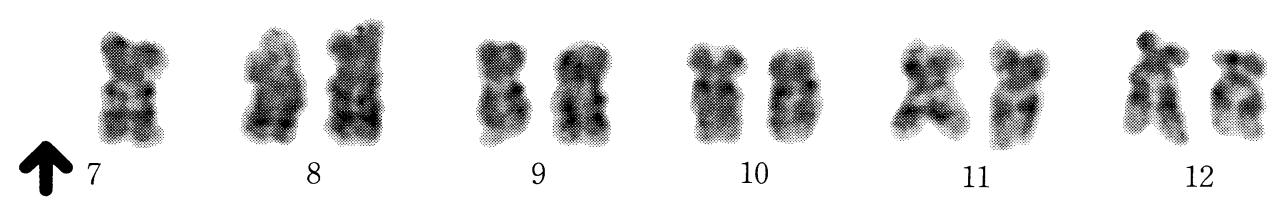

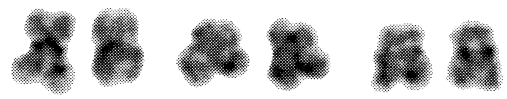

16

17

18

15

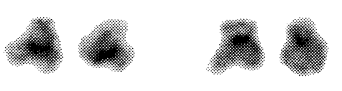

21

22

$\mathrm{X}$

Figure 2. Karyotype of Giemsa banded metaphase cell from unstimulated bone marrow of the patient. Cytogenetic analysis was performed on August 31, 1994, showing 45,XY,-7. 


\section{Evolution of SAA to MDS}

hemopoietic progenitor cells in the peripheral blood in SAA patients (9). These findings indicate that G-CSF and EPO treatment together with immunosuppression may be an effective means of therapy for SAA patients who do not have an HLA-match sibling.

A proportion of patients with AA who receive immunosuppressive treatment eventually develop a clonal disease, MDS or paroxysmal nocturnal hemoglobinuria, which may evolve into acute myeloid leukemia (10-12). This clonal evolution is likely due to the basic disease itself (13). The increased survival rate and the longer observation time period may explain the late hematological complications after immunosuppressive therapy. The evolution to MDS and leukemia has also been reported in SAA patients who have received G-CSF (14-16). Although the incidence of such hematological complications in AA patients who received G-CSF administration is not higher than that in reports without G-CSF treatment, all evolved cases with MDS and leukemia had cytogenetic abnormality monosomy 7 (15), as in the present case (Fig. 2). However, the clonal evolution with monosomy 7 has been reported in AA patients even before G-CSF became available (10). Further studies are needed to determine if G-CSF administration accelerates the clonal evolution in AA patients or if this cytogenetic abnormality is related to G-CSF administration.

\section{References}

1) Bacigalupo A, Hows J, Gluckman E, et al. Bone marrow transplantation (BMT) versus immunosuppression for the treatment of severe aplastic anaemia (SAA): a report of the EBMT SAA Working party. Br J Haematol 70: 177, 1988.

2) Kojima S, Fukuda M, Miyajima Y, Matsuyama T, Horibe K. Treatment of aplastic anemia in children with recombinant human granulocyte colony-stimulating factor. Blood 77: 937, 1991.

3) Ohsaka A, Kitagawa S, Yuo A, et al. Increased respiratory burst activity of neutrophils in patients with aplastic anemia: effects of granulocyte colony-stimulating factor and granulocyte-macrophage colony-stimulating factor. Exp Hematol 20: 1090, 1992.
4) Camitta BM, Thomas ED, Nathan DG, et al. A prospective study of androgens and bone marrow transplantation for treatment of severe aplastic anemia. Blood 53: 504, 1979.

5) Bessho M, Toyoda A, Itoh $Y$, et al. Trilineage recovery by combination therapy with recombinant human granulocyte colony-stimulating factor (rhG-CSF) and erythropoietin (rhEpo) in severe aplastic anaemia. Br J Haematol 80: 409, 1992.

6) Bertrand Y, Amri F, Capdeville R, French M, Philippe N. The successful treatment of two cases of severe aplastic anaemia with granulocyte colony-stimulating factor and cyclosporin A. Br J Haematol 79: 648, 1991.

7) Weide R, Lyttelton M, Samson D, et al. Sustained trilineage response in a patient with ALG-resistant severe aplastic anaemia after treatment with G-CSF, erythropoietin and cyclosporin A: Association of recovery with marked elevation of serum alkaline phosphatase. Br J Haematol 85: 608, 1993.

8) Ikebuchi K, Clark SC, Ihle JN, Souza LM, Ogawa M. Granulocyte colony-stimulating factor enhances interleukin 3-dependent proliferation of multipotential hemopoietic progenitors. Proc Natl Acad Sci USA 85: $3445,1988$.

9) Bacigalupo A, Piaggio G, Podestá M, et al. Collection of peripheral blood hematopoietic progenitors (PBHP) from patients with severe aplastic anemia (SAA) after prolonged administration of granulocyte colonystimulating factor. Blood 82: 1410, 1993.

10) De Planque MM, Kluin-Nelemans HC, van Krieken HJM, et al. Evolution of acquired severe aplastic anaemia to myelodysplasia and subsequent leukaemia in adults. Br J Haematol 70: 55, 1988.

11) Tichelli A, Gratwohl A, Würsch A, Nissen C, Speck B. Late haematological complications in severe aplastic anaemia. Br J Haematol 69: 413, 1988

12) Socié G, Henry-Amar M, Bacigalupo A, et al. Malignant tumors occurring after treatment of aplastic anemia. N Engl J Med 329: 1152, 1993.

13) Marsh JCW, Geary CG. Annotation: Is aplastic anaemia a pre-leukaemic disorder? Br J Haematol 77: 447, 1991

14) Kojima $S$, Tsuchida M, Matsuyama T. Myelodysplasia and leukemia after treatment of aplastic anemia with G-CSF. N Engl J Med 326: 1294 , 1992.

15) Imashuku S, Hibi S, Nakajima F, et al. A review of 125 cases to determine the risk of myelodysplasia and leukemia in pediatric neutropenic patients after treatment with recombinant human granulocyte colony-stimulating factor. Blood 84: 2380, 1994.

16) Izumi $T$, Muroi $K$, Takatoku $M$, Imagawa $S$, Hatake $K$, Miura $Y$. Development of acute myeloblastic leukaemia in a case of aplastic anaemia treated with granulocyte colony-stimulating factor. $\mathrm{Br} \mathrm{J} H$ Hematol 87: 666, 1994. 